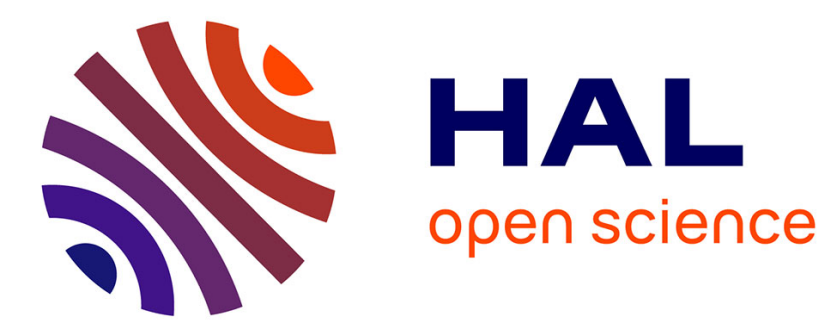

\title{
Fluorescence Correlation Spectroscopy
}

\author{
Patrick Ferrand, Jérôme Wenger, H. Rigneault
}

\section{To cite this version:}

Patrick Ferrand, Jérôme Wenger, H. Rigneault. Fluorescence Correlation Spectroscopy. Erwin J.G. Peterman and Gijs J.L. Wuite. Single Molecule Analysis, Methods and Protocols, Humana Press, Springer, pp.181-195, 2011, Methods in Molecular Biology, 10.1007/978-1-61779-282-3_10 . hal00624663

\section{HAL Id: hal-00624663 \\ https://hal.science/hal-00624663}

Submitted on 23 Apr 2015

HAL is a multi-disciplinary open access archive for the deposit and dissemination of scientific research documents, whether they are published or not. The documents may come from teaching and research institutions in France or abroad, or from public or private research centers.
L'archive ouverte pluridisciplinaire HAL, est destinée au dépôt et à la diffusion de documents scientifiques de niveau recherche, publiés ou non, émanant des établissements d'enseignement et de recherche français ou étrangers, des laboratoires publics ou privés. 


\title{
Fluorescence Correlation Spectroscopy
}

\author{
Patrick Ferrand, Jérôme Wenger and Hervé Rigneault, \\ Institut Fresnel \\ CNRS, Aix-Marseille Université, Ecole Centrale Marseille \\ Domaine universitaire de St Jérôme \\ 13397 Marseille, France \\ Email : patrick.ferrand@fresnel.fr
}

January 7, 2010

\begin{abstract}
Fluorescence Correlation Spectroscopy (FCS), implemented in microscopy, relies on performing an autocorrelation of the time fluctuating intensity arising from individual molecules diffusing through a confocal volume. It allows to investigate a large variety of dynamic processes and to quantify photophysical, photochemical, interaction, diffusion, and transport properties of molecules. This tutorial chapter is intended to give an "hands on" view of FCS. After a brief introduction on the principle of FCS, the major theoretical assumptions are emphasized, and the main analytical expression are given. Then the key parameters that have to be considered when building a FCS system are discussed. The complete method of operation is described, including calibration, measurement, and data treatment. The major difficulties that are encountered when performing for the first time FCS are illustrated by examples of measurements, and possible solutions are proposed.
\end{abstract}

Keywords: Fluorescence correlation spectroscopy, FCS, autocorrelation, fluctuations, single molecule spectroscopy, confocal microscopy, diffusion. 


\section{Introduction}

"Intensity is not enough", that could be the motto for Fluorescence Correlation Spectroscopy (FCS) a quite mature technique nowadays that was born in the 70's (1) from the work done at that time on dynamic light scattering (DLS) (2). FCS (as DLS) relies on performing a time correlation on a fluctuating light signal with the assumption that this fluctuating signal is coming from a limited and fluctuating number of particles, the size of the particles being small compared to the characteristic length of the observation volume. In principle time correlation can be performed on any kind of signal, we shall further be interested in fluorescence as the contrast mechanism used in FCS, whereas DLS relies on light scattering.
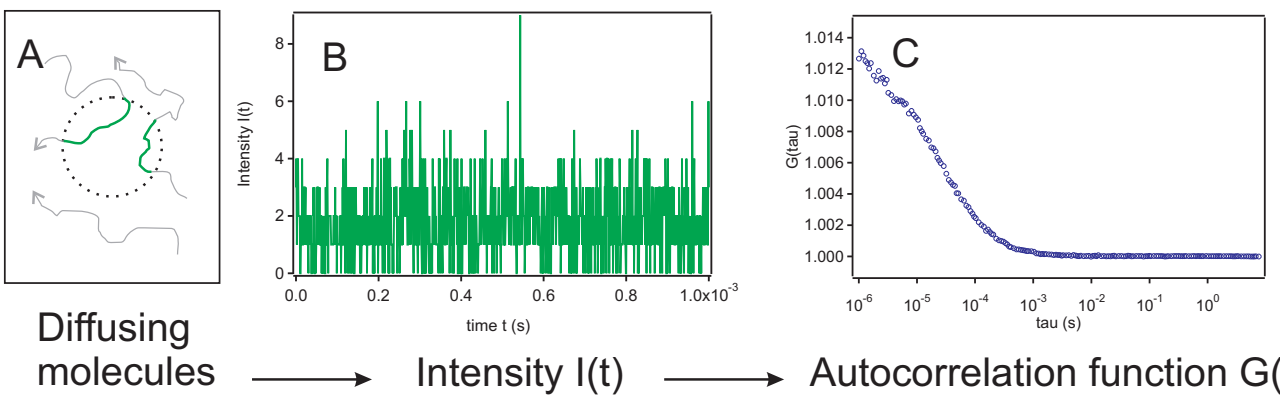

molecules

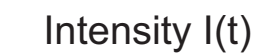

Autocorrelation function $\mathrm{G}(\mathrm{tau})$

Figure 1: Schematic view of a typical FCS measurement. A: Diffusion of fluorescent molecules through the observation volume of a confocal microscope (represented by the dotted circle). B: The resulting fluctuating fluorescence intensity. C: The autocorrelation function calculated from the intensity, according to Eq. 1.

To make things clear, let's consider a volume of observation, that defines a restricted area in a much larger open volume, where particles are freely moving, as illustrated in Fig. 1-A. For simplicity, we consider here a 2D diffusion process, this can be for instance what happens when a fluorescent molecule is freely moving in a biomembrane. Now looking at the fluorescence intensity recorded as a function of time in the observation volume, it is clear that molecule entering and living the volume will make this intensity fluctuate, as depicted in Fig. 1-B.

Now in FCS, we are not only interested in the amplitude of these fluctuations, but also in their average time duration. In principle, one could imagine 
retrieving this information by measuring how much time was elapsed between an increase and a decrease of fluorescence intensity. However, this view is very simplistic and in practice, such information is very difficult to extract, because there is no way to ensure that the obtained increase and decrease are due to the same molecule. On another hand, although the signal looks chaotic, it is obvious that any molecule entering the volume will leave after a certain time, on average. Indeed, the key of the the mathematical treatment of FCS relies on this on average notion, more generally on the concept of correlation. Starting from intensity $I(t)$, the so-called autocorrelation function $(\mathrm{ACF}) G(\tau)$ is defined as

$$
G(\tau)=\frac{\langle I(t) I(t+\tau)\rangle}{\langle I(t)\rangle^{2}}
$$

where the brackets \langle\rangle indicate a time average. The ACF has to be understood as a way to quantify how much a signal is still similar to itself after a given delay $\tau$. In our example of $2 \mathrm{D}$ diffusion, it is clear that the signal doesn't have the time to change too much within a delay $\tau$ if $\tau$ is much smaller than the typical residence time of the molecules in the observation volume. Mathematically, this would give $\langle I(t) I(t+\tau)\rangle \simeq\left\langle I^{2}(t)\right\rangle>\langle I(t)\rangle\langle I(t)\rangle$ and therefore $G(\tau)>1$. On the contrary, if $\tau$ is much larger than the typical residence time, then signals $I(t)$ and $I(t+\tau)$ have no similarity and $\langle I(t) I(t+$ $\tau)\rangle$ can be written as $\langle I(t)\rangle\langle I(t+\tau)\rangle$, thus $G(\tau)=1$. It is intuitive at this stage that the intermediate values of the ACF will carry the information on the duration of the fluctuation, here the residence time of the molecule in the observation volume. A typical ACF is plotted in Fig. 1-C. Although sensitive to single molecule fluctuation, FCS is intrinsically a statistic method, thanks to the huge number of individual events that are analyzed.

Although the above explanation is hand waving and limited to the simple case of 2D diffusion, for tutorial purposes, FCS is supported by a rigorous theory as we will see below. It permits to quantify a wide range of phenomena such as photophysical, photochemical, interaction, diffusion, and transport properties of molecules $(\mathbf{3})$.

The scope of this chapter is very practical, "what shall I do to build and run my own FCS setup" has been our guideline when writing this chapter. 


\section{$1.1 \quad$ Theoretical assumptions}

The most important assumption behind FCS measurements deals with the concept of stationarity. The system under study is assumed to be stationary, i. e., from a mathematical point of view, both quantities $\langle I(t)\rangle$ and $\langle I(t) I(t+\tau)\rangle$ of Eq. 1 need to be independent on time $t$. In other words, the total measurement duration $T_{\text {tot }}$ has to be much larger than the fluctuation duration (4). A practical figure of merit of the stationarity is the value of $G(\tau)$, that should be unity for the largest values of $\tau$. As we will see later, a signal that is not stationary is not be suitable for a FCS analysis.

The second assumption deals with the shape of the observation volume, that is described by the point spread function (PSF) of the confocal microscope. In the case of a high numerical aperture microscope objective, the PSF is in principle a complicated distribution that depends both on the excitation and collection optics. However, for FCS studies, it is generally assumed that it can be reasonably described by a 3D gaussian distribution (5)

$$
\operatorname{PSF}(x, y, z)=I_{0} \exp \left[-2\left(\frac{x^{2}+y^{2}}{\omega_{x y}^{2}}+\frac{z^{2}}{\omega_{z}^{2}}\right)\right],
$$

where $w_{x y}$ and $w_{z}$ denote the transverse and axial waist, respectively. With this assumption, an effective observation volume is usually defined as

$$
V_{\mathrm{eff}}=\pi^{3 / 2} w_{x y}^{2} w_{z}
$$

Although this Gaussian assumption does not constitute a sine qua non condition for performing FCS, it allows to derive relatively simple analytical expressions for the autocorrelation function $G(\tau)$, as we will see in section 1.5.

\subsection{Amplitude of the ACF}

By writing the fluctuating intensity as $I(t)=\langle I\rangle+\delta I(t)$, Eq. 1 becomes

$$
G(\tau)=1+\frac{\langle\delta I(t) \delta I(t+\tau)\rangle}{\langle I\rangle^{2}}
$$

In case of diffusing molecules, the fluctuations of intensity recorded by an ideal setup are only due to the fluctuation number of molecule in the observation volume. We shall name $\delta N$ this fluctuation number. For a given count rate per molecule (CRM), $\langle I\rangle=\langle N\rangle \cdot \mathrm{CRM}$ and $\delta I(t)$ can be simply 
written as $\delta I(t)=\delta N(t) \cdot \mathrm{CRM}$, and then, for short delays, the amplitude of the $\mathrm{ACF}$ is

$$
G(\tau \rightarrow 0)=1+\frac{\left\langle\delta N^{2}\right\rangle}{\langle N\rangle^{2}}=1+\frac{1}{\langle N\rangle},
$$

where we have used the property that the quantity $N$ is governed by a Poisson statistics, and as such, $\left\langle\delta N^{2}\right\rangle=\langle N\rangle$. The important result of Eq. 3 emhasizes that FCS is intrinsically designed to analyze small numbers of molecules.

\subsection{Relevant time scales}

Although FCS can address a large range of time scales, a priori knowledge of the fluctuation time, that we denote $\tau_{f}$, is necessary to set the optimum conditions for measurement, that we summarize here as

$$
\Delta \tau \ll \tau_{f} \ll T_{\text {tot }},
$$

where $\Delta \tau$ is the correlator sampling time with which the intensity $I(t)$ is recorded. Indeed, in order to be sensitive to the fluctuations, $\Delta \tau$ needs to be much smaller than $\tau_{f}$. Note that $\Delta \tau$ will be the first channel of the computed ACF, usually named the correlogram. In addition, as discussed in section 1.1, the condition of stationarity requires that the total measurement duration $T_{\text {tot }}$ is much larger than $\tau_{f}$. Note that $T_{\text {tot }}$ will be the last channel of the correlogram.

\subsection{Signal to noise ratio in FCS}

Noise and statistical accuracy in FCS deserve specific consideration. First, understanding the origin of the signal to noise ratio (SNR) enables optimization of the experimental apparatus. Second, proper weighting procedures based on statical estimates for the noise enable more accurate analysis. Beyond technical noise, two physical phenomena bring fundamental contributions to the noise in FCS : the quantum nature of light that induces shot noise, and the stochastic nature of the fluorescence fluctuation process itself.

In the most common experimental conditions for FCS: Gaussian molecular detection efficiency, 3D Brownian diffusion, negligible background, and small sample time $(\Delta \tau \rightarrow 0)$, but without any assumption on the average 
number of molecules $\langle N\rangle$, it can be demonstrated that the SNR of the autocorrelation function is simply given by $(\mathbf{6})$

$$
\mathrm{SNR}_{\tau \rightarrow 0} \simeq \frac{\mathrm{CRM} \sqrt{T_{\mathrm{tot}} \Delta \tau}}{(1+1 /\langle N\rangle)^{1 / 2}}
$$

where CRM denotes as previously the count rate per molecule. This shows that the SNR in FCS does not depend on the total detected fluorescence, but on the fluorescence count rate per molecule (CRM), on the square root of the experiment duration $T_{\text {tot }}$ and on the correlator channel minimum width $\Delta \tau$. This further emphasizes the single molecule nature of FCS, and provides guidelines to improve the statistical accuracy in FCS. For a fixed experimental apparatus, one can either increase the excitation power to raise the CRM, or wait for longer integration times $T_{\text {tot }}$. However, both of these strategies have significant practical limitations. First, saturation and photobleaching limit the CRM increase to a certain extent. Second, due to the square root dependence of the SNR on the acquisition time, increasing $T_{\text {tot }}$ to a few hundred seconds has only a minor influence on the SNR.

\subsection{Analytical expression of the ACF}

With the assumptions of section 1.1, analytical expressions of the ACF can be derived for a large variety of physical processes. For the sake of generality, we shall write the ACF as

$$
G(\tau)=1+\frac{1}{\langle N\rangle} X(\tau) G_{\mathrm{D}}(\tau) B(\tau)
$$

where $X(\tau), G_{\mathrm{D}}(\tau)$ and $B(\tau)$ denote the intra- or intermolecular reactions, diffusion, and background contributions, respectively, to the ACF. Note that this separation of the different dynamical contribution is only possible when molecular reactions takes place at much shorter time scale than diffusion, which is usually a reasonable assumption.

\subsubsection{Inter- and intramolecular dynamics}

The first factor in Eq. 6 accounts for changes in fluorescence yield as molecules undergo inter- or intramolecular dynamics, such as triplet blinking. The general expression for $X(\tau)$ is

$$
X(\tau)=1+\frac{T}{1-T} \exp \left(-\frac{\tau}{\tau_{\mathrm{T}}}\right)
$$


where $T$ is the fraction of molecule in a dark state, and $\tau_{\mathrm{T}}$ is the corresponding duration of the triplet fluorescence fluctuation (7).

\subsubsection{Translational diffusion}

The factor $G_{\mathrm{D}}(\tau)$ is derived from the diffusion equation that governs the evolution of the concentration $C(\mathbf{r}, t)$ of the fluorescent molecule, given by

$$
\frac{\partial C(\mathbf{r}, t)}{\partial t}=D \Delta C(\mathbf{r}, t)
$$

where $D$ is the diffusion coefficient of the molecule. For a $3 \mathrm{D}$ translational diffusion,

$$
G_{\mathrm{D}}^{3 \mathrm{D}}(\tau)=\frac{1}{1+\frac{\tau}{\tau_{D}}} \frac{1}{\sqrt{1+s^{2} \frac{\tau}{\tau_{D}}}}
$$

with $\tau_{D}=\frac{w_{x y}^{2}}{4 D}$ is the average residence time in the observation volume and $s=\frac{w_{x y}}{w_{z}}$ is a purely geometrical factor. In case of $2 \mathrm{D}$ diffusion the limit $s \rightarrow 0$ gives

$$
G_{\mathrm{D}}^{2 \mathrm{D}}(\tau)=\frac{1}{1+\frac{\tau}{\tau_{D}}}
$$

\subsubsection{Background signal}

Last but not least, the factor $B(\tau)$ accounts for the contribution of background signal in the ACF. It has been demonstrated that (6)

$$
B(\tau)=\left(1-\frac{\langle B\rangle}{\langle I\rangle}\right)^{2},
$$

where $\langle B\rangle$ is practically the intensity measured by replacing the fluorescent sample by a non fluorescent one.

\section{Materials}

\subsection{FCS system}

Several companies offer turn-key FCS systems. Most of them are based on confocal laser scanning microscope (CLSM) systems (see Note 1), while some 
others rely on a more simple optical systems, require no optical alignment but suit therefore to more diluted systems. These systems include excitation laser, appropriate filter sets, photon counting systems, and a dedicated data analysis software.

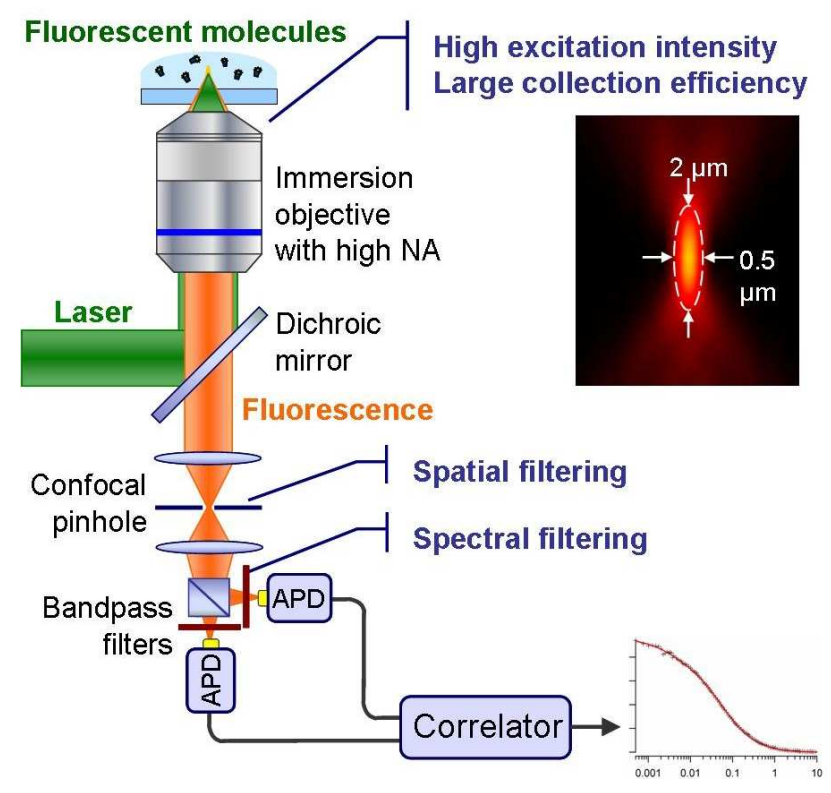

Figure 2: A basic FCS setup, as well as a typical correlogram as computed by the correlator. The inset shows a schematic view of the 3D PSF of the system. APD stands for avalanche photodiode.

Alternatively, most commercial CLSMs can be customized in order to perform FCS measurements, according to the optical scheme presented in Fig. 2. It requires however a solid background in photonics instrumentation, electronics, data acquisition and programming. The following points need to be considered

1. It is important to ensure that the scanning system can be turned off in one way or an other, in order to perform static measurements. Sometimes, this can be done by setting the scanning range to 0 .

2. A system with various available pinhole sizes will be useful is case of signal concerns (the larger the pinhole, the stronger the signal). 
3. A high numerical aperture objective (typically $\mathrm{NA}=1.2$ ) is preferable, because it ensures the largest collection efficiency and therefore the largest CRM, in addition to the highest spatial resolution.

4. Laser excitation power will need to be measured in order to provide controlled and producible operating conditions. Photodiode power meters are usually the best choice for low power measurement, i. e., below a few $\mathrm{mW}$.)

5. Because CLSM detection usually relies on photomultiplier tubes in analog mode, the detector must be replaced by one (or two, see Note 2) photon counting module(s) such as avalanche photodiodes (APD) (for instance, SPCM-AQR series by PerkinElmer Optoelectronics, PDM series by Micro-Photon-Devices, or id100 series by id Quantique). The key parameter to be considered here is quantum efficiency, because this directly affects the CRM.

6. The correlation function can be built up either by using a digital hardware correlator (by correlator.com or ALV-GmbH), or by software correlation. Although it means an additional cost, the first approach is the most easy to implement and also the most secure concerning numerical error and/or artifacts. In this case, the signal form the detectors is correlated on-line, and the ACF can be monitored in real time. Such correlator can work either in linear mode or in multi-tau mode, the later offering a wider range of accessible fluctuation times, thanks to channels of increasing width in a quasi logarithmic progression. Alternatively, correlation can be performed afterwards on the intensity series, usually in multi-tau or equivalent computation scheme. The major drawback of this approach is the huge volume of data that needs to be stored and the often prohibitive calculation time. For this reason, it is only relevant for slow processes (fluctuation time larger than $1 \mathrm{~ms}$ ). Even if the correlation is built up on-line, we recommend to always record (with a very low time sampling, typically $100 \mathrm{~ms}$ ), the time series, for further analysis of stationarity.

7. Last but no least, FCS data analysis strongly relies on advanced fitting procedures. The work flow will be much more effective by using data analysis software offering powerful fitting algorithms, such as 
Levenberg-Marquardt, as well as a complete macro programming language, such as Origin, Igor Pro, Matlab, etc.

\subsection{Labeling}

When choosing the fluorescent label to be used on the biological system you want to investigate, remember that the golden rule is "to maximize the CRM". Therefore,

1. Select robust dyes, labels, fluorescent proteins with the highest quantum efficiency,

2. Check carefully the filter set in order to match the spectral features of the label,

3. Always prefer labels that have been extensively documented in the literature. Even if they are not the subject of your investigations, photophysical properties such as conformal changes, triplet blinking, may be visible on the $\mathrm{ACF}$, and an a priori knowledge will by a real plus when fitting the data. Diffusion coefficient of the molecule in solution is required if the label is used to calibrate the measurement volume of your FCS system.

\subsection{Calibration solution}

The calibration solution will be used in order to determine the measurement volume of the system.

1. Choose a solution of a robust dye those fluorescence can be measured with the same laser/filter configuration as the one that labels your biological system.

2. Choose a dye those diffusion coefficient $D_{\text {cal }}$ is documented (8).

3. Feel free to use the same label as for your biological system, if you think it is stable and bright enough.

4. First prepare a 1-mL aliquot of high concentration in ultrapure water, typically $1 \mathrm{mM}$. This will constitute the stock solution for future dilutions. Keep it stored at $-18^{\circ} \mathrm{C}$. 
5. Prepare from the stock solution four successive dilutions in 1-mL aliquots with typical concentrations $10 \mu \mathrm{M}, 1 \mu \mathrm{M}, 100 \mathrm{nM}, 10 \mathrm{nM}$. Store at $+4^{\circ} \mathrm{C}$ in the dark.

\section{Methods}

\subsection{Coarse optical alignment}

Note that although CLSM can produce relative clear images even in case of a moderate misalignment, FCS is optically much more demanding and requires

- a perfect centering of the excitation laser on the back aperture of the microscope objective,

- a perfect conjugation of the pinhole with the excitation spot.

In case of a commercial FCS system or of a FCS-upgraded CLSM, some of the alignment settings may not be accessible to the user, and therefore require maintenance by the company. Note that this procedure needs to be followed only if the system has not been used for several weeks or if an important modification has been made on the optical system (laser replacement, filter change, etc.).

1. Switch the system on, with the correct excitation sources, microscope objective and filter set.

2. Select the largest pinhole diameter.

3. Stop the beam with an appropriate beam stopper. Set laser power to a moderate level. This should be a few hundreds $\mu \mathrm{W}$ for a onephoton fluorescence process and a few tens of $\mathrm{mW}$ for a two-photon fluorescence process.

4. Put a droplet of the appropriate immersion liquid on the objective.

5. Place a standard coverslip on the sample stage.

6. Put about $50 \mu \mathrm{L}$ of the most concentrated calibration solution $(10 \mu \mathrm{M})$.

7. Release the laser beam stopper. 
8. Move the focus until the laser spot is located inside the solution. You should see the fluorescence spot when looking at the sample, your eyes been protected from laser radiation by goggles. Alternatively, remove the sample holder, and place the coverslip directly on the objective, the capillary effect of the immersion should maintain it horizontally. This ensures that the observation volume is into the solution).

9. Switch on the detector. Run a static intensity measurement. Reduce laser excitation power if the detector is saturating.

10. Optimize the signal by adjusting the pinhole alignment. Reduce laser excitation power if the detector is saturating.

11. Repeat optimization with the next dilution $(1 \mu \mathrm{M})$.

12. Repeat optimization with a smaller pinhole diameter.

\subsection{Calibration of the measurement volume}

This procedure has to be followed before any series of measurements, in order to prevent any slight misalignment. In addition, it allows to determine the values of $w_{x y}$ and $w_{z}$ for the system. Steps $12-13$ allow to set the best measurement conditions for a given solution on the system. It does not need to be repeated daily.

1. Switch system on, with the appropriate excitation sources, microscope objective and filter set.

2. Select the largest pinhole diameter.

3. Set laser power to a moderate level. Stop the beam with a an appropriate beam stopper.

4. Put a droplet of the appropriate immersion liquid on the objective.

5. Place a standard coverslip on the sample stage.

6. Put about $50 \mu \mathrm{L}$ of the $100-\mathrm{nM}$ calibration solution.

7. Release the laser beam stopper.

8. Move the focus until the laser spot is located inside the solution. 
9. Record a ACF for $T_{\text {tot }}=30 \mathrm{~s}$, and measure simultaneously the average value of the fluorescence intensity $\langle I\rangle$. If ACF is too noisy, use a larger measurement duration.

10. Fit the obtained ACF with analytical expression of Eq. 6, in order to obtain values $\langle N\rangle, \tau_{D}$, as well as $T$ and $\tau_{T}$, if necessary. Usually, $s$ can be fixed to $s=0.2$ for the first fitting iterations.

11. From $\langle I\rangle$ and $\langle N\rangle$, calculate the count rate per molecule $\mathrm{CRM}=$ $\langle I\rangle /\langle N\rangle$.

12. Repeat steps 9 to 11 for various sizes of pinhole (see Notes 3 and 4) and various excitation powers $P_{\text {exc }}$.

13. Plot variations of CRM and $\tau_{D}$ versus $P_{\text {exc }}$. The range of excitation power for which CRM has a linear dependence upon $P_{\text {exc }}$ and $\tau_{D}$ does not depends on $P_{\text {exc }}$ is the one which suits to this calibration solution on your system. In practive, use a power $20 \%$ below the maximum suitable excitation power.

14. The lateral width of the measurement volume is given by $w_{x y}=\sqrt{4 D_{\text {ref }} \tau_{D}}$, where $\tau_{D}$ is measured in the correct range of excitation power.

An example of FCS performed in a $30 \mathrm{nM}$ solution of Rhodamine $6 \mathrm{G}$ in water (9), under a typical excitation power of $200 \mu \mathrm{W}$ is plotted in Fig. 3, as well as the corresponding fit, according to Eqs. 6, 7 and 8. Note that background signal was negligible and therefore $B(\tau)$ was assumed to be unity. Given a value $D_{\text {ref }}=280 \mu \mathrm{m}^{2} / \mathrm{s}$, measurement volume here is charactarized by $w_{x y}=179 \mathrm{~nm}$ and $w_{x y}=895 \mathrm{~nm}$.

\subsection{FCS measurements}

1. Place your sample on the microscope.

2. Set the excitation power to a moderate level.

3. In the case of a cell or other specific sample, use the LSCM capabilities of the system in order to set the correct focus and set the FCS measurement area (by moving the sample, or by moving the measurement spot, depending on the system in use). 


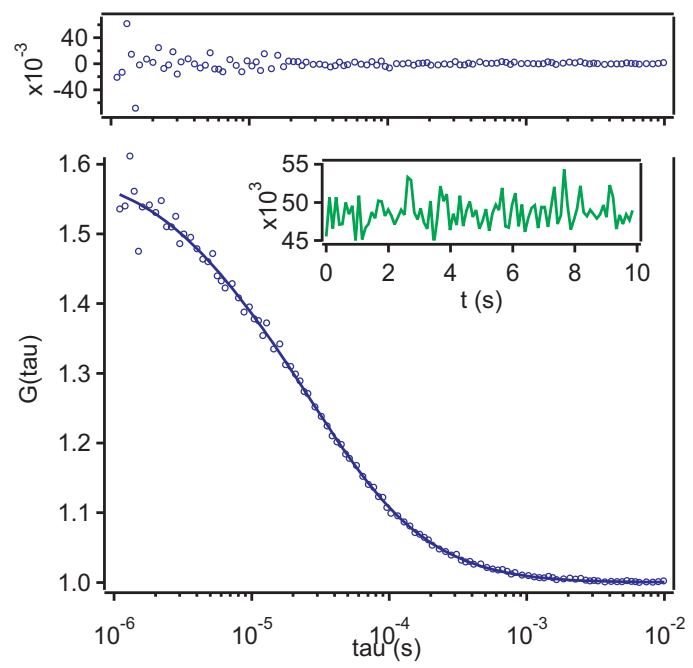

Figure 3: Example of ACF recorded in a calibration solution of Rhodamine 6G. Measurement duration was $10 \mathrm{~s}$. Circle are experimental points, while solid line is the fit according to Eqs. 6, 7 and 8. Inset is the intensity series, top graph is the residuals. Fitting values: $\langle N\rangle=1.93, T=0.133$, $\tau_{\mathrm{T}}=4.1 \mu \mathrm{s}, \tau_{\mathrm{D}}=29 \mu \mathrm{s}$, and $s=0.2$.

4. Set the excitation power to a low level, compatible with static recording of intensity (see Note 5).

5. Record a set of short correlogram, as well as the corresponding intensity series.

6. Repeat the measurement on equivalent locations, on another part of the sample.

\subsection{FCS data analysis}

1. Browse all correlograms together with the corresponding intensity series that have been recorded at one location.

2. Discard all correlograms whose intensity series are not stationary (see Note 6). This often happens in complex samples such as cells because of membrane fluctuations, dye aggregation, etc. 
3. Average the remaining correlograms.

4. Fit the obtained correlogram with the most appropriate model. If the obtained physical parameters do not make physical sense, reject the fit.

5. Repeat this procedure for all sets of correlograms.

\section{Notes}

1. FCS is not limited to one-photon absorption fluorescence, but has been successfully extended to non-linear optical contrasts, such as twophoton absorption fluorescence, etc. From an instrumental point of view, because non-linear microscopy systems are free of pinhole, detection relies usually on large area photomultiplier tubes. It is therefore subject to parasitic light, in addition to a lower quantum efficiency. In practice two-photon FCS is subject to strong photobleaching and requires significantly higher incident power (tens of $\mathrm{mW}$ ) than onephoton FCS (hundred of $\mu \mathrm{W}$ ). Nevertheless it has several advantages like being able to excite several fluorophores with the same wavelength; it doesn't require pinhole, it has a potential deeper penetration depth in scattering samples.

2. Detectors can be subject to measurement artifacts (dead time, after pulse, etc.), that compromise the ACF for short delays (typically for $\tau<1 \mu \mathrm{s})$. In case values at short delays are necessary for the process under study (photophysics for instance), this random noise can be efficiently rejecting by splitting the signal on two detectors, and by cross-correlating the signals obtained by the two detectors. The system illustrated in Fig. 2 is set up in this configuration.

3. Pinhole diameter is directly related to the axial extent of the PSF. Although this feature is crucial for CLSM imaging, because it determines the axial sectioning capability of the system, it can often be reasonably sacrificed while doing FCS measurement, a larger pinhole diameter providing a significantly larger CRM, and therefore a larger SNR. Indeed, enlarging the pinhole diameter produces only limited modifications of the diffusion time $\tau_{D}$. This is because $w_{x y}$ (transverse width of observation volume) is defined by the excitation beam rather than the collection optics (that defined $w_{z}$ ). 


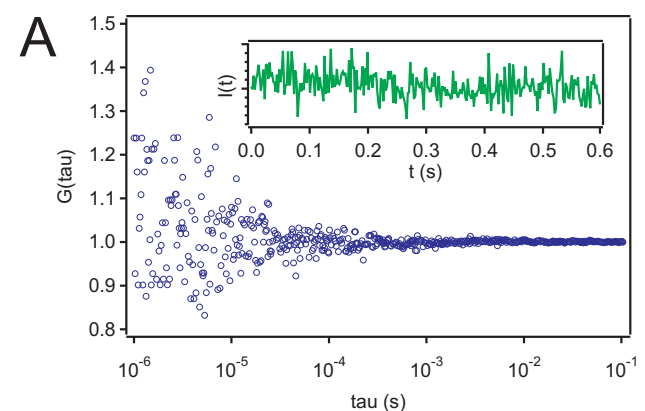

Problem

- No visible correlation

Diagnosis

- Concentration is too high

- Sample is out of focus

- Sample is static

To do

- Use a sample with less fluorophores

- Check focus
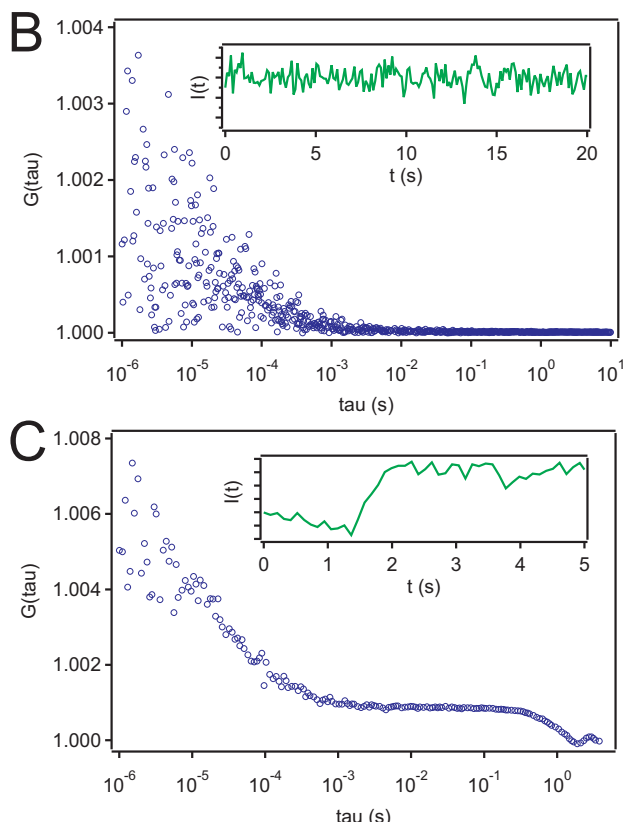

Problem

- Low SNR

Diagnosis

- CRM is too low

- Measurement duration is too low

To do

- Improve CRM (increase excitation power, use a

larger pinhole, increase excitation power)

- Change fluorophore for a brighter one

- Change the microscope objective for one with a

higher NA

Problem

- Intensity is not stationary

- Shape of correlogram changed suddently

Diagnosis

- sample drift

- intercept of a large aggregate

To do

- Try to purify your sample

- Replace long duration measurement by several short duration measurement, discard odd correlograms and average

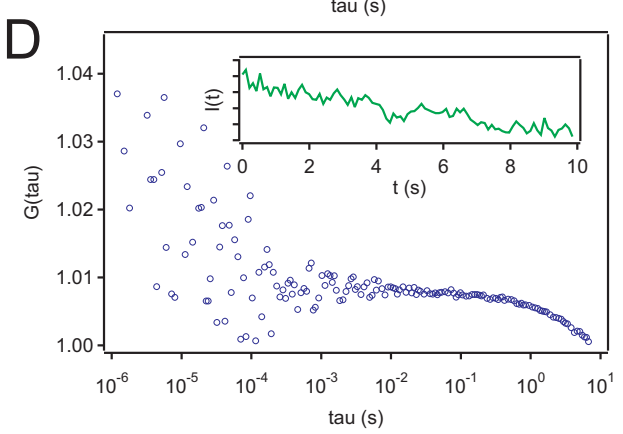

Problem

- Intensity is dropping continuously

- Correlogram "flies away"

Diagnosis

- photobleaching of the static or slow fraction of the sample

To do

- Use a lower excitation power

- If you are only interested in the fast moving fraction,

also consider the possibility to i) bleach out all the

slow fraction, ii) run your FCS measurement on the

remaining fast fraction.

Figure 4: A non exhaustive panel of unsuitable correlograms, with the corresponding intensity series plotted in the inset. For each case the problem is detailed, diagnosis and possible solutions are given. 
4. When repeating measurements with new samples, you may experience a sudden degradation of CRM and/or diffusion time, these two figures being of course much more clearly visible when measuring solutions. This is often due to a defect of the coverslip, that is sometimes outside the nominal range indicated by the manufacturer. In this case, it is usually more effective to discard the coverslip and to start a new measurement than to try to adjust the correction collar of the microscope objective. Another common problem is the drying of water between the coverslip and a water-objective lens.

5. Although FCS can address fluctuation processes at all timescales (within the limitations mentioned in section 1.3, of course), slow diffusion cannot be measured in practice, because slow species are usually bleached before leaving the measurement volume. This produces a typical drop of the recorded intensity, as illustrated in Fig. 4-D. In this case, scanning FCS approaches such as image correlation spectroscopy and its variants may be more relevant (see Note 7).

6. Fig. 4 illustrates some examples of odd-looking correlograms that constitute the usual first taste of FCS for new users and that may be quite discouraging. Possible explanations and suggestions are given for each case. Measurements like cases $\mathrm{C}$ and $\mathrm{D}$ must be definitely discarded, because they do not satisfy to the condition of stationarity required by the FCS analysis. Although these correlograms may in some cases be successfully fitted, they would produce erroneous values. More generally, these examples emphasize that FCS analysis must be operated by experienced users only, especially for measurements carried out in complex systems such as cells, where the diffusion is mostly non Brownian and the signal subjected to other spurious fluctuations.

7. Latest development of FCS focus both on hardware and software. From the hardware side the current trends are (i) dual spot measurement to measure FCS simultaneously at various locations (9) and/or to access to absolute diffusion coefficient (8), (ii) multispot FCS using EMCCD cameras as detector (10). From the data analysis side, recent development focus on the improvement of spatio-temporal information by means of scanning FCS (11) and Image correlation spectroscopy techniques (12) that give access to both temporal and spatial correlations. Another powerful variant of FCS is Fluorescence Cross Correlation 
Spectroscopy (FCCS), which extends the capabilities of standard FCS by introducing two different fluorescent probes with distinct excitation and/or emission properties, which can be detected in the same confocal volume. It allows to probe interactions between different molecular species (13).

\section{References}

[1] Magde, D., Elson, E. L., and Webb, W. W. (1972) Thermodynamic fluctuations in a reacting system measurement by fluorescence correlation spectroscopy. Phys. Rev. Lett. 29, 705.

[2] Berne, B. J., and Pecora, R. Dynamic light scattering: with applications to chemistry, biology, and physics; Dover, 2000.

[3] Webb, W. W. (2001) Fluorescence correlation spectroscopy: inception, biophysical experimentations, and prospectus. Appl. Opt. 40, 3969.

[4] Tcherniak, A., Reznik, C., Link, S., and Landes, C. F. (2008) Fluorescence correlation spectroscopy: criteria for analysis in complex systems. Anal. Chem. 81, 746.

[5] Rigler, R., Mets, U., Widengren, J., and Kask, P. (1993) Fluorescence correlation spectroscopy with high count rate and low-background analysis of translational diffusion. Eur. Biophys. J. 22, 169.

[6] Wenger, J., Gérard, D., Aouani, H., Rigneault, H., Lowder, B., Blair, S., Devaux, E., and Ebbessen, T. W. (2009) Nanoaperture-enhanced signalto-noise ratio in fluorescence correlation spectroscopy. Analytical chemistry 81, 834-9.

[7] Widengren, J., Mets, U., and Rigler, R. (1995) Fluorescence correlation spectroscopy of triplet-states in solution - a Theoretical and experimental-study. J. Phys. Chem. 99, 13368.

[8] Müller, C. B., Loman, A., Pacheco, V., Koberling, F., Willbold, D., Richtering, W., and Enderlein, J. (2008) Precise measurement of diffusion by multi-color dual-focus fluorescence correlation spectroscopy. Europhys. Lett. 83, 46001. 
[9] Ferrand, P., Pianta, M., Kress, A., Aillaud, A., Rigneault, H., and Marguet, D. (2009) A versatile dual spot laser scanning confocal microscopy system for advanced fluorescence correlation spectroscopy analysis in living cell. Rev. Sci. Instr. 80, 083702.

[10] Burkhardt, M., and Schwille, P. (2006) Electron multiplying CCD based detection for spatially resolved fluorescence correlation spectroscopy. Opt. Express 14, 5013.

[11] Petrášek, Z., Hoege, C., Mashaghi, A., Ohrt, T., Hyman, A., and Schwille, P. (2008) Characterization of protein dynamics in asymmetric cell division by scanning fluorescence correlation spectroscopy. Biophys. J. 95,5476 .

[12] Kolin, D. L., and Wiseman, P. W. (2007) Advances in image correlation spectroscopy: Measuring number densities, aggregation states, and dynamics of fluorescently labeled macromolecules in cells. Cell Biochem. Biophys. 49, 141.

[13] Bacia, K., Kim, S. A., and Schwille, P. (2006) Fluorescence crosscorrelation spectroscopy in living cells. Nature Meth. 3, 83. 